\title{
Is immunogenetic susceptibility to neuropsychiatric systemic lupus erythematosus (SLE) different from non-neuropsychiatric SLE?
}

\author{
Lucienir M Silva, Eduardo A Donadi
}

\begin{abstract}
Objectives-To analyse frequency of HLA class II antigens (DR and DQ) and lymphocytotoxic autoantibodies in patients with systemic lupus erythematosus (SLE) and subsets with or without neuropsychiatric involvement.
\end{abstract}

Methods-Ninety three patients with SLE (42 with neuropsychiatric features) were typed for HLA class II antigens and investigated for the presence of lymphocytotoxic autoantibodies by a complement dependent microlymphocytotoxicity assay. A total of 191 controls of similar ethnic background were also typed for HLA antigens.

Results-HLA-DR3 antigen was increased in the total group of patients with SLE $(p=0.003)$ and in the neuropsychiatric group $(p=0.002)$. HLA-DR4 antigen frequency was increased in nonneuropsychiatric patients $(p=0.001)$ and decreased in patients with neuropsychiatric SLE $(p=0.0005)$. Comparisons of HLA frequencies between subgroups of patients showed decreased HLA-DR4 ( $p<0.0001)$ and increased HLA-DR9 and HLA-DQ2 antigens $(p=0.0008$ and 0.005 respectively) in the neuropsychiatric group. The frequency of lymphocytotoxic autoantibodies was increased in neuropsychiatric patients with SLE having HLA-DR9 specificity $(p=0 \cdot 04)$.

Conclusion-HLA-DR4 may have a protective specificity for the development of neuropsychiatric features of SLE and HLA-DR9, in addition to HLA-DR3, and the presence of lymphocytotoxic autoantibodies may predispose to neuropsychiatric abnormalities.

Immunology

Department of

Internal Medicine,

School of Medicine of

Ribeirão Preto,

University of São

Paulo, 14049-900,

Ribeirão Preto, São

Paulo, Brazil

L M Silva

E A Donadi

Correspondence to:

Dr Eduardo A Donadi,

Division of Clinical

Immunolgy, Department of

Internal Medicine, School of

Medicine of Ribeirão Preto,

University of São Paulo,

Avenida Bandeirantes, 3900

14049-900, Ribeirão Preto,

São Paulo, Brazil.

Accepted for publication

17 April 1996

(Ann Rheum Dis 1996; 55: 544-547)

Systemic lupus erythematosus (SLE) is a complex multisystemic disease associated with neuropsychiatric abnormalities in more than $50 \%$ of patients. ${ }^{1}$ The entire nervous system may be affected, with symptoms and signs ranging from focal to diffuse. Generalised seizures, psychosis, transverse myelitis, focal seizures, stroke, peripheral and cranial neuropathies, aseptic meningitis, and movement disorders have been described as characteristic features of neuropsychiatric lupus (NPSLE). ${ }^{2}$ Because NPSLE may occur in the absence of generalised disease and in view of the virtual absence of histopathologically documentable vasculitis within the brain, ${ }^{34}$ there is controversy as to whether the pathogenesis of NPSLE is different from that of SLE without neuropsychiatric involvement. Autoantibodies that react with neuronal cell membranes, lymphocytotoxic antibodies that cross react with brain tissue, and antibrain antibodies that cross react with lymphocytes have been implicated in the pathogensis of NPSLE. ${ }^{5-7}$ On the other hand, lymphocytotoxic serum from patients with SLE exhibits reactivity against $\beta 2$-microglobulin and HLA class I and II molecules. ${ }^{8} 9$ To explore whether immunogenetic susceptibility to NPSLE is different from SLE without neuropsychiatric features we investigated HLA class II antigens in these SLE subsets. We also evaluated the possible association of HLA antigens and lymphocytotoxic autoantibodies.

\section{Patients and methods}

PATIENTS

We studied 93 patients with SLE, diagnosed according to the criteria of the American College of Rheumatology, ${ }^{10}$ and seen at the University Hospital of the School of Medicine of Ribeirão Preto, Brazil, from 1991 to 1994. Forty two patients had neuropsychiatric features classified according to a workshop consensus. ${ }^{2}$ There were 29 white patients (all of them females), six Afro-European (five females and one male), and seven black patients (six females and one male). Their median age was 34 (range 13-66) years. Fifty one patients did not have neuropsychiatric features, 36 were white ( 35 female, one male), nine were Afro-European, and six were black with a median age of 30 (range 10-53) years.
Total lymphocytes were isolated using a FicollHypaque gradient at a density of $1.077 \mathrm{~g} / \mathrm{l}$. B lymphocytes were obtained by adherence to nylon wool (Robbins Scientific, USA); HLA

antisera against DR1-DR18, DR52-DR53,

CONTROL GROUP

A total of 191 healthy blood donors from the same geographic area $(79 \%$ white, $12 \%$ AfroEuropean, and $9 \%$ black), were typed for HLA class II antigens. A total of 30 healthy subjects were also assayed for lymphocytotoxic autoantibodies. 
and DQ1-DQ7 antigens were obtained from Pel Freez, Gene Trak, and Fred Hutchinson Cancer Research Centre (USA), and Biotest (Germany). Serological HLA class II typing was performed with a complement dependent microlymphocytotoxicity assay. ${ }^{11}$

\section{LYMPHOCYTOTOXIC AUTOANTIBODIES}

Blood was drawn from patients with either neuropsychiatric or non-neuropsychiatric active disease. Lymphocytotoxic antibody was detected using the patient's own lymphocytes in a complement dependent microlymphocytotoxicity assay. ${ }^{11}$ Incubations were performed at room temperature and lymphocytotoxins were considered to be present when there was at least $20 \%$ cell lysis.

\section{STATISTICAL ANALYSIS}

A two tailed exact Fisher's test was used for comparisons of HLA frequencies, with corrections of the $p$ values according to the number of specificities tested and the number of comparisons made. Differences were considered to be significant at $p<0 \cdot 05$. Relative risk (RR), which indicates how many times more often the disease occurs in people with the HLA antigen compared with those without it, aetiological fraction (EF), which indicates

Table 1 Clinical and laboratory features of patients with SLE and subsets

\begin{tabular}{llll}
\hline & $\begin{array}{l}\text { Neuropsychiatric SLE } \\
(n=42) \\
n(\%)\end{array}$ & $\begin{array}{l}\text { Non-neuropsychiatric SLE } \\
(n=51) \\
n(\%)\end{array}$ & $\begin{array}{l}\text { Total group SLE } \\
(n=93) \\
n(\%)\end{array}$ \\
\hline Nephritis & $36(85 \cdot 7)$ & $30(58 \cdot 8)$ & $66(70 \cdot 6)$ \\
Arthritis & $32(76 \cdot 2)$ & $47(92 \cdot 1)$ & $79(84 \cdot 9)$ \\
Malar rash & $17(40 \cdot 5)$ & $25(49)$ & $42(45 \cdot 1)$ \\
Photosensitivity & $16(38 \cdot 1)$ & $21(41 \cdot 2)$ & $37(39 \cdot 7)$ \\
Pleuritis & $15(35 \cdot 7)$ & $16(31 \cdot 4)$ & $31(33 \cdot 3)$ \\
Oral ulcers & $11(26 \cdot 2)$ & $3(5 \cdot 9)$ & $14(15)$ \\
Discoid rash & $9(21 \cdot 4)$ & $15(29 \cdot 4)$ & $24(25 \cdot 8)$ \\
Pericarditis & $8(19 \cdot 1)$ & $10(19 \cdot 6)$ & $18(19 \cdot 3)$ \\
Antinuclear antibodies & $40(95 \cdot 2)$ & $50(98 \cdot 1)$ & $90(96 \cdot 7)$ \\
Lymphopenia & $32(76 \cdot 2)$ & $35(68 \cdot 2)$ & $67(74)$ \\
Anti-dsDNA & $21(50)$ & $13(25 \cdot 5)$ & $34(36 \cdot 5)$ \\
Anti-Ro/SSA & $15(35 \cdot 7)$ & $19(37 \cdot 2)$ & $34(36 \cdot 5)$ \\
Lymphocytotoxic antibodies & $12(28 \cdot 5)$ & $1(1 \cdot 9)$ & $13(13 \cdot 9)$ \\
Anti-Sm & $10(23 \cdot 8)$ & $13(25 \cdot 5)$ & $23(24 \cdot 7)$ \\
False positive test for syphilis & $9(21 \cdot 4)$ & $9(17 \cdot 6)$ & $18(19 \cdot 3)$ \\
Anticardiolipin antibodies & $5(11 \cdot 9)$ & $4(7 \cdot 9)$ & $9(9 \cdot 6)$ \\
\hline
\end{tabular}

Table 2 Frequency (\%) of HLA-DR and HLA-DQ antigens in patients with neuropsychiatric SLE, patients with non-neuropsychiatric SLE, total SLE group, and control subjects

\begin{tabular}{|c|c|c|c|c|c|}
\hline Antigen & $\begin{array}{l}\text { Neuropsychiatric } \\
S L E(n=42) \\
(\%)\end{array}$ & $\begin{array}{l}\text { Non-neuropsychiatric } \\
\text { SLE }(n=51) \\
(\%)\end{array}$ & $\begin{array}{l}\text { Total group } \\
\text { SLE } \\
(\%)\end{array}$ & $\begin{array}{l}\text { Control } \\
(n=191) \\
(\%)\end{array}$ & pc Value \\
\hline DR1 & $21 \cdot 4$ & $21 \cdot 6$ & 21.5 & 29 & NS \\
\hline DR2 & $26 \cdot 2$ & $21 \cdot 6$ & $23 \cdot 6$ & $26 \cdot 2$ & NS \\
\hline DR3 & $47 \cdot \overline{6}$ & $41 \cdot 2$ & $44 \cdot 1$ & 23 & $0.003^{\star}$ \\
\hline DR4 & 9.5 & $64 \cdot 7$ & 39.8 & $36 \cdot 6$ & NS \\
\hline DR5 & $28 \cdot 6$ & $21 \cdot 6$ & $24 \cdot 7$ & $23 \cdot 6$ & NS \\
\hline DR6 & $7 \cdot 1$ & $9 \cdot 8$ & $8 \cdot 6$ & $13 \cdot 1$ & NS \\
\hline DR7 & 11.9 & $7 \cdot 8$ & $9 \cdot 7$ & $13 \cdot 1$ & NS \\
\hline DR8 & - & - & - & $2 \cdot 1$ & NS \\
\hline DR9 & $26 \cdot 2$ & 1.9 & $12 \cdot 9$ & $13 \cdot 1$ & NS \\
\hline DR10 & - & - & - & 0.5 & NS \\
\hline DR 11 & $7 \cdot 1$ & 3.9 & 5.4 & $16 \cdot 3$ & NS \\
\hline DR13 & - & - & - & 1.5 & NS \\
\hline DR14 & $7 \cdot 1$ & $9 \cdot 8$ & $8 \cdot 6$ & $5 \cdot 7$ & NS \\
\hline DR52 & $78 \cdot 6$ & $74 \cdot 5$ & $76 \cdot 4$ & $74 \cdot 3$ & NS \\
\hline DR53 & $42 \cdot 8$ & 54.9 & 49.5 & $57 \cdot 1$ & NS \\
\hline DQ1 & $52 \cdot 4$ & $66 \cdot 7$ & $60 \cdot 2$ & $66 \cdot 5$ & NS \\
\hline DQ2 & $21 \cdot 4$ & 1.9 & $10 \cdot 7$ & $8 \cdot 4$ & NS \\
\hline DQ3 & $73 \cdot 8$ & $86 \cdot 3$ & $80 \cdot 6$ & 86.9 & NS \\
\hline DQ7 & $28 \cdot 6$ & $31 \cdot 4$ & $30 \cdot 1$ & $36 \cdot 6$ & NS \\
\hline
\end{tabular}

$\star$ Relative risk $=2 \cdot 65$; aetiological fraction $=0 \cdot 27$.

The $\mathrm{p}$ value refers to the comparison of total group of patients $v$ controls. how much the HLA marker contributes to disease at the population level, and preventive fraction (PF), which is an indicator of protection, were also estimated. ${ }^{12}$

\section{Results}

The most frequent neuropsychiatric features were organic brain syndrome $(33 \cdot 3 \%)$, psychosis $(30.9 \%)$, generalised seizures $(30.9 \%)$, cranial neuropathies $(28.6 \%)$, stroke $(23 \cdot 8 \%)$, peripheral neuropathies (19.1\%; half associated with other disorders of the central nervous system and half isolated), aseptic meningitis $(9.5 \%)$, pseudotumour cerebri $(7 \cdot 1 \%)$, and transverse myelitis $(4 \cdot 8 \%)$. About $62 \%$ of patients presented neuropsychiatric manifestations considered to be of diffuse disease (organic brain syndrome, generalised seizures, psychosis), associated or not with focal disease, and $38 \%$ had only manifestations considered to be of focal disease (transverse myelitis, aseptic meningitis, pseudotumour cerebri, and cranial and peripheral neuropathies). Table 1 shows the additional clinical and laboratory features of the whole group of patients.

Table 2 shows the frequencies of HLA class II antigens. Specificity for HLA-DR3 was significantly increased in the total group of patients with SLE $(p=0.003)$. Compared with controls, the patients with NPSLE also presented a significantly increased HLA-DR3 antigen ( $p=0.002 ; \quad R R=3.03 ; \quad E F=0.31)$. Compared with controls, HLA-DR4 specificity was overrepresented in patients with SLE without neuropsychiatric features $(p=0.001$; $\mathrm{RR}=3 \cdot 16 ; \mathrm{EF}=0.44)$ and underrepresented in patients with NPSLE ( $p=0.0005$; $R R=0 \cdot 18 ; P F=0.52)$. Comparisons of HLA class II frequency between patients with and without neuropsychiatric features showed an increased frequency of HLA-DR9 and HLADQ2 $(p=0.0008$ and $p=0.005$ respectively) and a decreased frequency of HLA-DR4 antigens $(p<0.0001)$ in patients with NPSLE. In patients with NPSLE, the comparisons between diffuse or focal disease with controls or with each other did not disclose significant differences in HLA antigen frequency.

Lymphocytotoxic autoantibodies were detected in 12 of 38 patients with NPSLE with involvement of the central nervous system and in only one of 20 randomly selected patients with non-neuropsychiatric SLE $(p=0 \cdot 02)$. All controls were negative. Of the patients with lymphocytotoxic autoantibodies five were HLA-DR9 (all NPSLE), and eight were nonHLA-DR9 (seven NPSLE and one nonNPSLE). Therefore, the presence of lymphocytotoxic autoantibodies was positively associated with the presence of HLA-DR9 $(p=0.04)$. Only one patient had both lymphocytotoxic autoantibody and HLA-DQ2 antigen $(p=0 \cdot 2)$. There were no associations between HLA antigens in NPSLE and other laboratory variables shown in table 1 .

Compared with controls, the frequency of the combination of antigens HLA-DR3/DR4 was increased in non-NPSLE patients 
$(p=0.003)$ and HLA-DR3/DR9 was increased in patients with NPSLE $(p=0.04)$.

\section{Discussion}

Although patients with SLE and controls were from varied ethnic backgrounds, the frequency of white, Afro-European, and black people, and the frequency of HLA antigens in these ethnic groups did not differ significantly between patients and controls. This study showed that HLA-DR3 antigen was also increased in the total group of patients with SLE, in agreement with a previous study. ${ }^{13}$ There are few studies correlating HLA antigens and clinical and laboratory defined subsets of SLE. In our series, the HLA-DR3 antigen conferred susceptibility to, whereas HLA-DR4 conferred protection against the development of NPSLE. As HLA-DR4 antigen has been previously associated with protection against the development of renal SLE, ${ }^{14}$ and most $(85 \cdot 7 \%)$ of our patients with NPSLE presented also nephritis, the question of whether renal SLE could act as a confounder should be considered. For our total group of patients, the frequency of HLA-DR4 antigen in patients with nephritis and controls $(42 \cdot 4 \%$ and $36 \cdot 6 \%$ respectively) was not significantly different (uncorrected $p=0.46$ ). In addition, HLA-DR4 frequency did not differ significantly (uncorrected $\mathrm{p}=0.35$ ) between patients with and without nephritis $(42.4 \%$ and $33.3 \%$ respectively). For combinations of SLE subsets (presence or absence of neuropsychiatric or renal involvement), the comparisons of HLADR4 frequency among these subsets did not disclose any significant association. Therefore, in this study, the negative association between HLA-DR4 antigen and NPSLE was not influenced by concomitant renal SLE.

In the search for immunogenetic features which could confer susceptibility to NPSLE, we compared HLA frequencies between two subsets of patients with SLE (with or without neuropsychiatric involvement). Both diffuse and focal NPSLE were lumped together because some pathogenetic features described for diffuse disease have also been shown in focal disease. ${ }^{7}$ An increased frequency of HLADR9 and HLA-DQ2 antigens has not been previously reported in NPSLE. A previous study conducted on Japanese patients with NPSLE showed an increased frequency of HLA-DR1 antigen. ${ }^{15} \mathrm{We}$ also found a positive association between lymphocytotoxic autoantibody and HLA-DR9 antigen. The following findings may support the role of autoantibodies in the pathogenesis of NPSLE, as well as the relation between lymphocytotoxic antibodies and HLA antigens. Compared with activity in serum, increased antineuronal antibody activity has been detected in the CSF of patients with NPSLE. ${ }^{7}$ Lymphocytotoxic antibodies present in the serum samples of patients with NPSLE have shown activity against neurons in the cortex, cerebellum, and caudate nucleus. ${ }^{5}$ Antibrain antibodies found in the serum of patients with NPSLE cross react with lymphocytes. ${ }^{6}$ Lymphocytotoxic antibodies react with lymphocyte antigens such as CD45RA (naive lymphocytes), $\beta 2$ microglobulin, and the heavy chain of HLA class I antigens, and HLA class II molecules. ${ }^{8}$ Because true vasculitis has been a rare pathological finding in NPSLE, ${ }^{34}$ lymphocytotoxic or antineuronal antibodies are probably causing disease by direct antibody mediated cellular damage or dysfunction. Finally, the presence of HLA-DR9 antigen in addition to HLA-DR3 may act as a suceptibility marker for the development of the neuropsychiatric features of SLE.

Few studies have investigated the presence of lymphocytotoxic antibodies bound to the lymphocytes of patients with SLE. ${ }^{16}$ In this study, we investigated only warm reactive lymphocytotoxic antibodies (reactive at room temperature), and we did not separate lymphocyte subsets to verify specific cytotoxicity. Warm reactive lymphocytotoxic antibodies have been shown to be of the IgG class, they may produce lymphopenia directly or by means of the increased production of interferon- $\gamma$, they seem to act like antinuclear antibodies, and have the highest toxicity in NPSLE. ${ }^{16}$ In our series, lymphopenia occurred in $76.2 \%$ of patients with NPSLE and in $68.2 \%$ of patients with SLE without neuropsychiatric features. About $30 \%$ of patients with NPSLE and lymphopenia had lymphocytotoxic autoantibodies compared with only about $7 \%$ of lymphopenic patients without NPSLE. Although a direct association between lymphopenia and lymphocytotoxic antibodies would be expected, studies reported in the medical literature have not shown such association, ${ }^{16}$ suggesting that additional pathogenetic mechanisms may also be involved.

In conclusion, patients with NPSLE do have peculiar immunogenetic markers. HLA-DR3, HLA-DR9 and HLA-DQ2 antigens were associated with susceptibility, whereas HLADR4 was associated with protection against the development of NPSLE. We also showed an association between HLA-DR9 antigen and the presence of lymphocytotoxic autoantibody. Further studies of the nucleotide sequences which encode the antigen binding groove of candidate susceptibility and protective molecules may identify regions involved in the presentation of peptides derived from relevant autoantigens, and contribute to the understanding of the pathogenesis of SLE.

1 Hughes G R V. Central nervous system lupus: diagnosis and treatment. $\mathcal{F}$ Rheumatol 1980; 7: 405-11

2 Singer J, Denburg J A, and The ad hoc Neuropsychiatric Lupus Workshop Group. Diagnostic criteria for neuropsychiatric systemic lupus erythematosus: the results of consensus meeting. F Rheumatol 1990; 17: 1397-402.

3 Johnson R T, Richardson E P. The neurological manifestations of systemic lupus erythematosus: a clinicalpathological study of 24 cases and review of literature. Medicine (Baltimore) 1968; 47: 337-69.

4 Ellis S G, Verity M A. Central nervous system involvemen in systemic lupus erythematosus: a review of neuropathological findings in 57 cases, 1955-77. Semin Arthritis Rheum 1979; 8: 212-21.

5 Bresnihan B, Oliver M, Williams B, Hughes G R V. An antineuronal antibody cross-reacting with erythrocytes antineuronal antibody cross-reacting wich erythrocytes and lymphocytes in systemic

6 Wilson H A, Winfield J B, Lahita R G, Koffler D. Association of IgG anti-brain antibodies with central nervous Arthritis Rheum 1979; 22: 458-62. 
7 Bluestein H G, Williams G W, Steinberg A D. Cerebrospinal fluid antibodies to neuronal cells: association with neuropsychiatric manifestations of systemic lupus erythematosus. Am $\mathcal{F}$ Med 1981; 70: 240-6.

8 Messner R D, De Horatius R, Ferrone S. Lymphocytotoxic antibodies in systemic lupus erythematosus patients and their relatives. Reactivity with the HLA antigenic molecular complex. Arthritis Rheum 1980; 23: 265-72.

9 Okudaira K, Searles R P, Ceuppens L J, Goodwin J S, Williams-Jr R C. Anti-Ia reactivity in sera from patients with systemic lupus erythematosus. $\mathcal{f}$ Clin Invest 1982; 69: with syst

10 Tan E M, Cohen A S, Ries J F, et al. The 1982 revised criteria for the classification of systemic lupus erythematosus. Arthritis

11 Terasaki P I, McClelland J, Park M S, McCurly B. Microdroplet lymphocyte cytotoxicity test. In: Ray J G, Hare D B, Pedersen P D, Kayhoe D E, eds. Manual of tissue typing techniques. Bethesda: NIH, 1974: 66-77.
12 Svejgaard A. HLA and disease. In: Rose N R, Friedman H, Fahey J L. eds. Manual of clinical laboratory immunology. Washington, DC: American Society for Microbiology, 1986: 912-20

13 Schur P H, Marcus-Bagley D, Awdeh Z, Yunis E J, Alper C A. The effect of ethnicity on major histocompatibility complex complement allotypes and extended haplotypes in patients with systemic lupus erythematosus. Arthritis Rheum 1990; 33: 985-92.

14 Fronek Z, Timmerman L A, Alper C A, et al. Major histocompatibility complex genes and susceptibility to histocompatibility complex genes and susceptibility to systemic lupus erythematosus. Arthritis Rheum 1990; 33:

15 Kawai T, Katoh K, Tani K, Okuda K, Okubo T. HLA antigens in Japanese patients with central nervous system lupus. Tissue Antigens 1990; 35: 45-6.

16 Osman C, Swaak A J G. Lymphocytotoxic antibodies in SLE: a review of the literature. Clin Rheumatol 1994; 13: 21-7. 